\section{Vocal Cord Dysfunction bei Kindern und Jugendlichen}

Zusammenfassung: Während der letzten Jahre wird der Fehlfunktion des Stimmbandes - insbesondere dem paradoxen inspiratorischen Glottisschluss (Vocal Cord Dysfunction oder VCD) vermehrt Aufmerksamkeit geschenkt. Obwohl die Krankheit schon seit 1983 bekannt ist und seitdem zumindest kasuistisch auch immer wieder in Erinnerung gebracht wurde, wird die Diagnose häufig versäumt und die Fehldiagnose eines Asthma bronchiale gestellt - mit zum Teil deletären Folgen für die Betroffenen. Der inspiratorische Glottisschluss führt zu einer akut einsetzenden stridorösen Atmung mit akuter Dyspnoe, so dass die differenzialdiagnostische Annahme eines Asthmaanfalles durchaus nahe liegend ist. Diskriminierend sind häufig der inspiratorische Stridor und der perakute Ablauf, die Zweifel an der Diagnose Asthma wecken müssen. Es wurden in dieser Zusammenstellung von Kasuistiken die Symptome und die diagnostischen Möglichkeiten der VCD dargestellt und diskriminierende Befunde zum Asthma bronchiale werden betont. Wir berichten über fünf Kinder mit VCD (4 Mädchen, 1 Junge) im Alter von 2 bis 13 Jahren. Es kann an diesen Patienten deutlich gemacht werden, dass der VCD ein multifaktorieller Pathomechanismus zugrunde liegt, der somatische und psychogene Aspekte hat. Überraschend war, dass bei allen Kindern eine pathologische gastroösophageale Refluxaktivität - zum Teil mit erheblichem Ausmaß - gefunden wurde, ohne dass typische Symptome einer gastroösophagealen Refluxkrankheit wie retrosternale Schmerzen, Bauchschmerzen oder rezidivierendes $\mathrm{Er}$ brechen vorlagen (sog. silente Refluxer). Die Vielfalt der zugrunde liegenden Pathomechanismen und die nicht selten vorliegende Koinzidenz mit einem allergischen Asthma macht einen für jeden Betroffenen individualisierten Therapieplan notwendig.

Vocal Cord Dysfunction in Children and Adolescents: More attention has been given to vocal cord dysfunction (VCD) in the past years. Even though the disease is known since 1983 and was brought to mind at least casuistically through the years, often VCD was not diagnosed as such, being mistaken for bronchial asthma - at times with grave consequences for the patient. VCD causes the acute onset of stridulous respiration with acute dyspnea making the differential diagnosis of asthma quite suggestive. The inspirational stridor and a peracute progression of the disease should cause doubts as to the diagnosis of asthma. We collected case reports and describe the symptoms and diagnostic approaches to VCD as well as showing dis-

Pneumologie 2001; 55: $378-384$

(c) Georg Thieme Verlag Stuttgart · New York ISSN 0934-8387

\section{P. Ahrens, Y. Seibt, R. Kitz}

Zentrum für Kinderheilkunde und Jugendmedizin der Universität Frankfurt,

Klinik für Kinderheilkunde I, Pädiatrische Pneumologie, Frankfurt/Main (Leiter: Prof. Dr. H. Böhles)

criminating findings towards bronchial asthma. We report on five children suffering from VCD ( 4 girls, 1 boy) aged from 2 to 13 years. It could be shown that VCD is caused by a multifactoral pathomechanism, which has both somatic and psychological aspects. A surprising find was that all of the children were diagnosed with a pathological gastroesophageal reflux, partly in serious dimensions, without showing the typical symptoms such as retrosternal pain, stomach-ache or recurrent vomiting (so called silent reflux). Since there is a multitude of pathomechanisms involved and the disease often coincides with allergic asthma, an individual therapy plan is needed for each patient.

\section{Einleitung}

Die paradoxe Funktion des Stimmbandes mit der Folge eines inspiratorischen Glottisschlusses (Vocal Cord Dysfunction) führt zu einem perakut einsetzendem Zustand von Atemnot mit überwiegend inspiratorischem Stridor. Es ist durch diesen Pathomechanismus leicht erklärbar, dass Bronchodilatatoren und Steroide keine Wirksamkeit zeigen können. Der paradoxe Glottisschluss wird genau wie auch der akute Asthmaanfall durch Triggerfaktoren induziert, zu denen körperliche Belastung, aggressive Gerüche, Allergene und auch Stress gehören. Die VCD erfüllt damit zumindest formal Kriterien, die auch für das Asthma kennzeichnend sind, so dass die Fehleinschätzung der Situation und damit die Fehlbehandlung des Patienten häufig ist [1-4]. Da die Anfälle von VCD in der Regel nach relativ kurzer Zeit - meist innerhalb von Minuten - spontan enden, entsteht der Eindruck, die Notfalltherapie des „Asthmaanfalles“ - habe den therapeutischen Erfolg herbeigeführt. Nicht selten werden so mehrfach monatlich hohe Steroiddosen appliziert, mit der Folge schwerster Nebenwirkungen [5]. Nicht selten werden die Patienten notfallmäßig intubiert oder sogar tracheotomiert [6]. Eine Diskriminierung von Asthma und einer VCD - bzw. auch das Erkennen einer Koinzidenz ist für den Patienten von größter Bedeutung.

Zur Inzidenz und Prävalenz der VCD gibt es nur wenige Daten. Für Kinder liegen keine Untersuchungen vor. Für Erwachsene wurde der Anteil einer VCD bei Patienten mit belastungsinduzierter Dyspnoe auf ca. 15\% bestimmt [7]. Betroffen sind überwiegend Frauen mit ca. 80\%. Bei Kindern sind Mädchen mit ca. $60 \%$ vertreten $[1,8-10]$. Aus der Tatsache einer deutlichen Überrepräsentierung von medizinischen Berufen, einem häufig zu beobachtenden höheren Bildungsgrad der VCD-Patienten und einer häufigen Begleitpsychopathologie wie übermäßige Leistungsorientierung, allgemeine Angststö- 
rungen. Bei Persönlichkeitsstörungen und Missbrauchserlebnissen wird auf einen relevanten psychogenen Hintergrund bei der Erkrankung geschlossen [11]. Aus den bisher publizierten Kasuistiken ergibt sich, dass etwa 75\% der erwachsenen VCD-Patienten koinzident an Asthma bronchiale leiden. Von Heatley [12] wird ein Säugling berichtet, dessen VCD durch Behandlung einer gastroösophagealen Refluxkrankheit mit Ranitidin und Metoclopramid innerhalb von 5 Wochen geheilt war. Wegen des für die Autoren „offensichtlich erkennbaren kausalen Zusammenhanges“ wird postuliert, dass zumindest bei einer Subgruppe der VCD-Patienten ein ursächliches Zusammenwirken anzunehmen ist.

\section{Symptome der VCD}

Leitsymptom ist der schwere Atemnotzustand in Kombination mit einem inspiratorischen Stridor sowie ein Druckgefühl im Kehlkopfbereich [8]. Berichtet wird darüber hinaus über Heiserkeit und Husten. Auslöser der Atembeschwerden ist oft körperliche Anstrengung [10,13,14]. Eindrucksvoll ist der plötzliche Beginn der Atemnot und häufig auch das ebenso abrupte Ende der Symptomatik.

\section{Diagnose der VCD}

Der Anamnese kommt eine wesentliche diagnoseweisende Funktion zu. Die Lungenfunktion ist im symptomfreien Intervall in der Regel unauffällig [3] - im akuten Anfall ist typischerweise der inspiratorische Teil der Fluss-VolumenKurve massiv abgeflacht und der Kurvenverlauf zeigt oft ein charakteristisches „Sägezahnmuster“ $[3,4]$. Eine Flusslimitierung wird aber gelegentlich auch exspiratorisch beobachtet. Besonders bei koinzidentem Asthma ergeben sich darüber hinaus noch die asthmatypischen Fluss-Volumen-Muster. Als hinweisend für eine VCD kann eine Diskrepanz zwischen forciertem ex- und inspiratorischem Fluss bei deutlich verminderter inspiratorischer Leistung erwartet werden [15].

Diagnostische Methode der Wahl ist die Laryngoskopie in Spontanatmung während eines Anfallsereignisses. Hierbei zeigt sich die Adduktion der Stimmbänder bei der Einatmung. Typischerweise liegen die Stimmbänder dann in den vorderen $2 / 3$ aneinander, lassen aber im hinteren Bereich oft eine dreieckige Öffnung frei, eine sog. „chink deformity“ $[2,16]$.

Eine weitere Möglichkeit des Nachweises einer VCD besteht in der ultrasonographischen Darstellung der Stimmbänder und der Taschenfalten. Die Methode ist nichtinvasiv und bietet daher besonders für Kinder Vorteile. Sie hat allerdings erhebliche Limitierungen, da eine exakte Darstellung aller zur Diagnosefindung wichtiger Strukturen häufig nicht gelingt [18].

\section{Diskriminierungsmöglichkeiten von VCD und Asthma}

Charakteristisch für die VCD ist der tagsüber auftretende, schlagartige, häufig belastungsgetriggerte Beginn der Dyspnoe. Selten wird von Patienten mit nächtlichen Beschwerden berichtet [18]. Diagnostisch wegleitend ist der ausgeprägte inspiratorische Stridor. Korrespondierend ergibt sich in der Fluss-Volumen-Kurve eine deutliche inspiratorische Flusslimitierung. Charakteristisch ist die fehlende Reaktion auf Gabe eines $\beta$-Sympathomimetikums. Patienten mit VCD haben in der Regel normale Blutgaswerte, nur selten kommt es zu einer leichten Hypoxämie [19].

Diagnostisch verwirrend ist die häufige Koinzidenz von Asthma und VCD, wobei die oben aufgeführten VCD-typischen Merkmale jedoch zumindest Hinweise liefern und damit den Verdacht auf eine asthmakoinzidente VCD lenken.

Seltene und daher nur kursorisch erwähnte Differenzialdiagnosen einer VCD sind vergrößerte Strukturen im Mediastinum (sog. „mediastinal mass syndrome“), Rekurrensparesen, eine akute Fremdkörperaspiration, gelegentlich bewegliche und temporär atemwegsverschließende Neoplasien oder Granulome sowie subglottische Ödeme im Rahmen toxischer, allergischer oder pseudoallergischer Reaktionen.

\section{Fallbeispiele}

\section{Patientin 1}

Die Patientin wurde wegen eines inspiratorischen Stridors mit 9 Jahren erstmals ambulant vorgestellt. Der Stridor fiel im Liegen nur geringgradig - im Stehen und bei Belastung jedoch heftig auf. Eine konstante schwere Dyspnoe mit inspiratorischem Stridor nach dem Sportunterricht führte dann zu einer stationären Aufnahme. Die allergologische Diagnostik ergab eine Sensibilisierung gegen Erle und Birke der RAST-Klasse I. Eine 24-Stunden-2-Punkt-pH-Metrie war am proximalen Messpunkt mit 16 Refluxen, einem Reflux von 14 Minuten Dauer und einer Fraction-Time von 2,2\% pathologisch. Distal, in klassisch gastroenterologischer Messposition der Sonde, ergab sich eine numerisch fast normalwertige Messung.

In der Laryngo-Bronchoskopie zeigte sich eine ausgeprägte Laryngotracheitis. Die Ösophagoskopie ergab deutlich entzündliche Veränderungen der ösophagealen Schleimhaut. Aufgrund dieser Befunde wurde eine antibiotische Therapie eingeleitet und mit topischem Steroid, Omeprazol und Cisaprid therapiert. Unter dieser Therapie ging der Stridor schnell zurück und trat nur noch gelegentlich für kurze Zeit wieder auf.

3 Monate nach dem Absetzen der Therapie traten erneut heftige tägliche Anfälle von Stridor und Dyspnoe auf. Die vorherige Therapie wurde wiederholt mit erkennbarer Verbesserung der Situation innerhalb eines Tages, erneut eingesetzt. Trotz Fortführung der Therapie kam es aber dann wieder vermehrt zu Problemen und häufigen Anfallsereignissen. Abb. 1a zeigt die VCD-typische-Fluss-Volumen-Kurve der Patientin während eines Anfalles. Abb.1b zeigt die FlussVolumen-Kurve im symptomfreien Intervall.

Die psychologische Exploration des Kindes führte zu dem Ergebnis einer sehr engen Mutter-Kind-Bindung und einer auffälligen gemeinsamen Symptomfixierung.

Nach einem Gespräch mit der Patientin und der Mutter, in dem ein Kuraufenthalt des Mädchens ohne Begleitung der Mutter vorgeschlagen und geplant wurde, sistierten die voher nicht behandelbaren Beschwerden erneut über mehrere Wochen, um nach dem Absetzen von Omeprazol in alter Heftigkeit erneut aufzutauchen. Seit der Gabe eines Omeprazol-Plazebos über 4 Wochen ist das Kind nunmehr beschwerdefrei. 

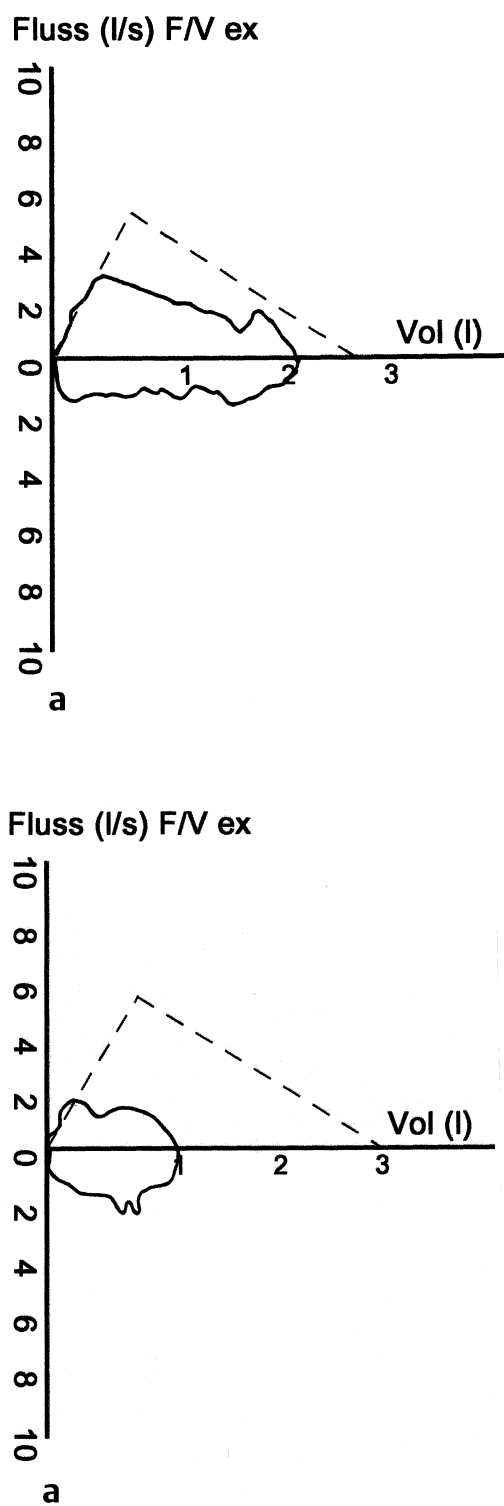

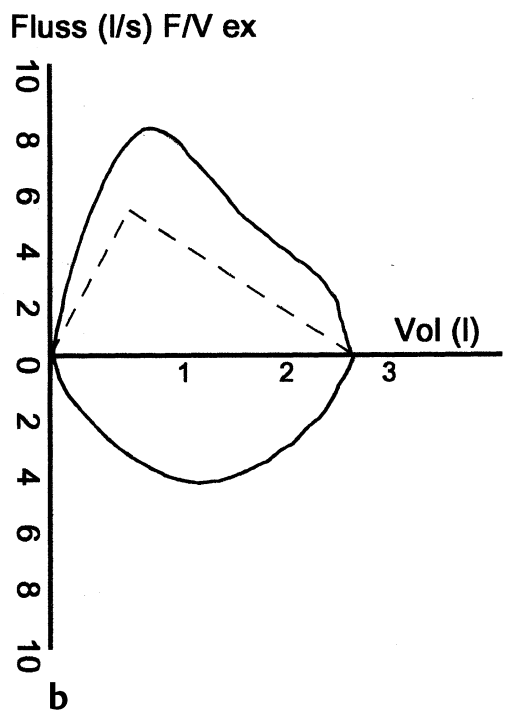

Abb. 1 a) Fluss-Volumen-Kurve (Pat. 1) während eines Anfalles. In- und exspiratorische Flusslimitierung bei inspiratorisch wellenförmigem Verlauf. b) Normale FlussVolumen-Kurve (Pat. 1) im beschwerdefreien Intervall.

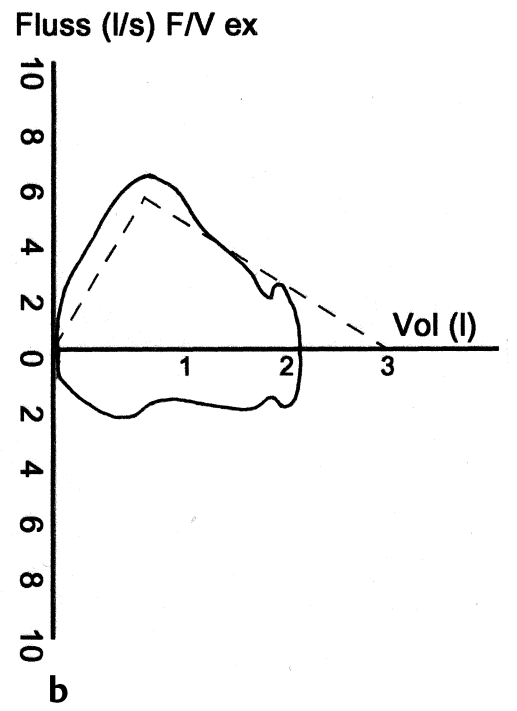

Abb. 2 a) Fluss-Volumen-Kurve (Pat. 2) während eines Anfalles. In- und exspiratorische Flusslimitierung bei inspiratorisch „sägezahnförmigem“ Verlauf. b) Normale Fluss-Volumen-Kurve (Pat. 2) nach Gabe von Xylocain-Spray.

\section{Patientin 2}

Die 13-jährige Patientin wurde wegen Atemnot, Druckschmerzen im Hals, und einem lauten Atemgeräusch notfallmäßig eingewiesen. Innerhalb der letzten zwei Wochen war dies das vierte ähnlich ablaufende Ereignis. Bei der Aufnahme konnte das Kind nicht sprechen und hatte einen laut hörbaren inspiratorischen Stridor. Die Sauerstoffsättigung wurde zwischen 96 und $98 \%$ gemessen.

In der Fluss-Volumen-Kurve zeigte sich bei einer belastungsprovozierten Reaktion der für die VCD typische Verlauf des Inspirationsflusses und eine ausgeprägte exspiratorische Flusslimitierung (Abb. 2a). Nach Applikation eines Sprühstoßes Xylocain sistierten die Beschwerden sofort (Abb. 2b).

Im Rahmen eines später auftretenden Anfalles kam es allerdings auch auf die Scheingabe von Xylocain-Spray (es wurde nur der Applikator in den Mund gehalten) zu einer sofortigen Beschwerdefreiheit. Auffällig waren ein IgE von $671 \mathrm{U} / \mathrm{ml}$ mit
Sensibilisierung gegen die Birke und die Milben. Die Provokationstestungen waren positiv. Ein Anfall konnte während der spezifischen Provokationen jedoch nicht induziert werden.

Eine über 14 Stunden durchgeführte 2-Punkt-pH-Metrie zeigte mit proximal 43 Refluxen eine deutlich erhöhte Refluxanzahl.

Bei der Laryngo-Bronchoskopie wurde der für die VCD typische Verschluss der Stimmritze bei der Inspiration unter Spontanatmung verifiziert.

Eine psychologische Untersuchung der Patientin ergab eine deutliche Überforderung durch die schulischen Ansprüche eines Gymnasiums, denen sie bei durchschnittlicher Intelligenz nur bedingt gewachsen war.

Die Option, Anfälle mit Xylocain zu durchbrechen, führte zu einer erfreulichen Stabilisierung der Patientin. Sie hatte zwar öfter Anfälle, die aber mit Xylocain-Spray sofort und leicht (selbst) zu behandeln waren. Stationäre Behandlungsnotwen- 

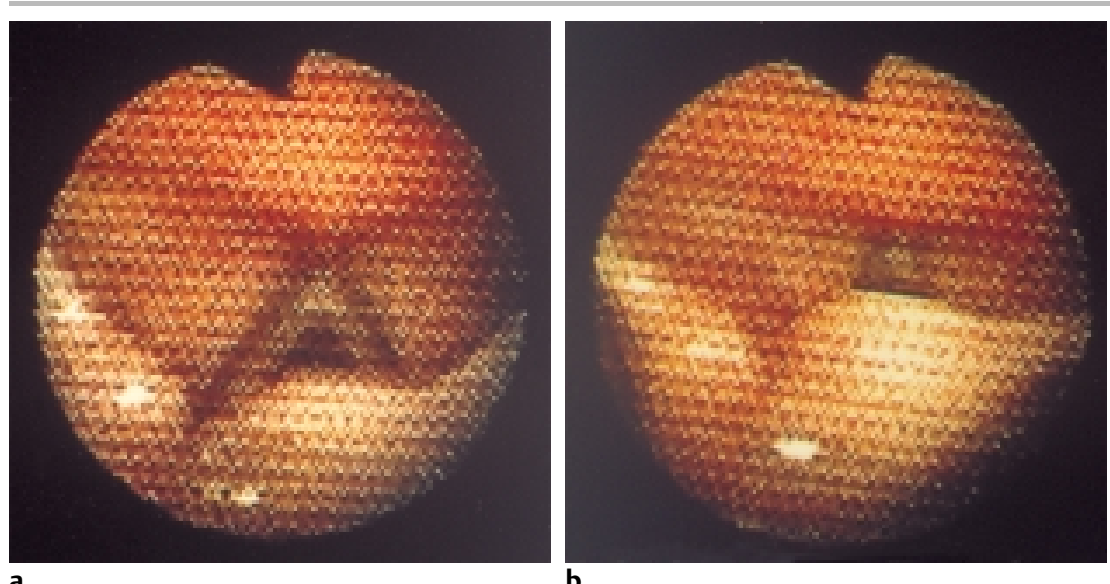

Abb. 3 a, b Glottisschluss bei Inspiration (Pat. 4): Zu Beginn der Inspiration ist eine weite Stimmritze zu erkennen (a), die sich im Verlauf der Inspiration deutlich verengt (b). Es ist darüber hinaus als Folge eines instabilen Larynx eine zentral gerichtete und damit das Lumen einengende Bewegung der Aryhöcker zu erkennen.

digkeit trat nach 6 Monaten erstmals wieder auf: Die Patientin wurde mit inspiratorischem Stridor notfallmäßig in die Ambulanz gebracht. Einfache Atemübungen (Stakkatoatmen gegen den ca. $15 \mathrm{~cm}$ entfernten Finger) führten $\mathrm{zu}$ einer raschen Symptomfreiheit.

\section{Patientin 3}

Die Zuweisung der 2 Jahre alten Patientin erfolgte wegen nachts auftretender Dyspnoe mit Zyanose und kruppähnlichem Husten. Sie hatte im Laufe der letzten 8 Wochen insgesamt 12-mal notfallmäßig hohe Dosen von Prednisolon (jeweils 30-100 mg) bekommen. Die stationäre Aufnahme erfolge in einem beschwerdefreien Zustand zur Abklärung. Die oMDP zeigt einen gastroösophagealer Reflux bis in das obere Ösophagusdrittel. In der 2-Punkt-pH-Metrie wurde für den proximalen Messpunkt ein hochpathologisches Ergebnis erhalten: bei 24 Refluxepisoden proximal wurde der längste Reflux hier mit 76 Minuten registriert. Die Laryngo-Bronchoskopie verifiziert den inspiratorischen Verschluss der Stimmritze. Das Kind hat darüber hinaus eine milde Tracheomalazie.

In der Ösophago-Gastroskopie imponieren Ösophagus und Magen völlig unauffällig. Es wurde eine Therapie mit Omeprazol und Cisaprid über 3 Monate durchgeführt sowie eine topische Steroiddauertherapie etabliert. Die Anfallsfrequenz und -intensität hat sich unter dieser Therapie deutlich reduziert. Zwischenzeitlich ist das Kind beschwerdefrei.

\section{Patientin 4}

Bei dem 11-jährigen Mädchen wurde eine schwere Dyspnoe mit inspiratorischem Stridor im Rahmen des Schulsportes induziert. Vorangegangen waren bereits häufig ähnliche Ereignisse mit weniger dramatischem Charakter.

Bei der Aufnahme zeigt das Mädchen eine deutliche Dyspnoe ohne Zyanose. Der inspiratorische Stridor sistiert nach Inhalation von Adrenalin und i.v. Gabe von Prednisolon. Die Blutgasanalyse bei der Aufnahme ist unauffällig.

Anamnestisch und im Prick-Test finden sich keine Hinweise für eine Allergie. Die Lungenfunktion im symptomfreien Intervall ist normal. Auch beim Belastungstest treten keine Symptome auf. Allerdings kommt es während des stationären Aufenthaltes wiederholt spontan zu dyspnoeisch-stridorösen
Ereignissen, die nach geringer Zeit spontan sistieren. Die pulsoxymetrische Überwachung während dieser Episoden zeigt keine Sättigungsabfälle.

Laryngoskopisch wurde im Anfall eine milde Laryngomalazie in Kombination mit einer inspiratorisch-paradoxen Stimmbandbewegung dokumentiert (Abb. 3a u.b). Eine oMDP ergibt einen spontanen gastroösophagealen Reflux bis in das obere Ösophagusdrittel. Die 2-Punkt-pH-Metrie zeigte proximal mit 31 Refluxen ein pathologisches Ergebnis. Hochpathologisch ist die distale Messung mit 234 Refluxen und einer FractionTime von $12,9 \%$.

Die Therapie mit Omeprazol führt zu keiner erkennbaren Reduktion der Anfallsfrequenz. Möglicherweise interessant im Zusammenhang mit einer Deutung des Krankheitsbildes auf einem psychogenen Hintergrund ist die Tatsache, dass die Patientinnen 4 und 2 Schülerinnen in der gleichen Schulklasse sind.

Beide führen erfolgreich zur Anfallsbewältigung Stakkatoatmung in der Schule durch.

\section{Patient 5}

Der 12-jährige Junge wurde von der Mutter wegen des nunmehr sechsten Anfalles eines inspiratorischen Stridors mit schwerer Atemnot und Husten in die Klinik gebracht. Die Mutter sieht einen Zusammenhang mit seelischen Belastungssituationen in der Schule. Zu Hause sei es bisher nie zu einem Anfallsereignis gekommen.

Bei der Aufnahme ist das Kind völlig unauffällig. Die Daten der Fluss-Volumen-Kurve sind regelrecht. Sensibilisierungen in den RAST-Testungen lagen nicht vor.

Die hochpathologische 2-Punkt-pH-Metrie ergibt proximal 35 Refluxe. Der längste Reflux wird hier mit 43 Minuten registriert. Ebenfalls pathologisch stellt sich die Messung in distaler Position dar (Abb.4). Die oMDP verifiziert einen gastroösophagealen Reflux bis in Jugulumhöhe.

Die psychologische Evaluierung ergibt eine deutlich verminderte Frustrationstoleranz und es wird eine Ablösungsproblematik erkennbar. 


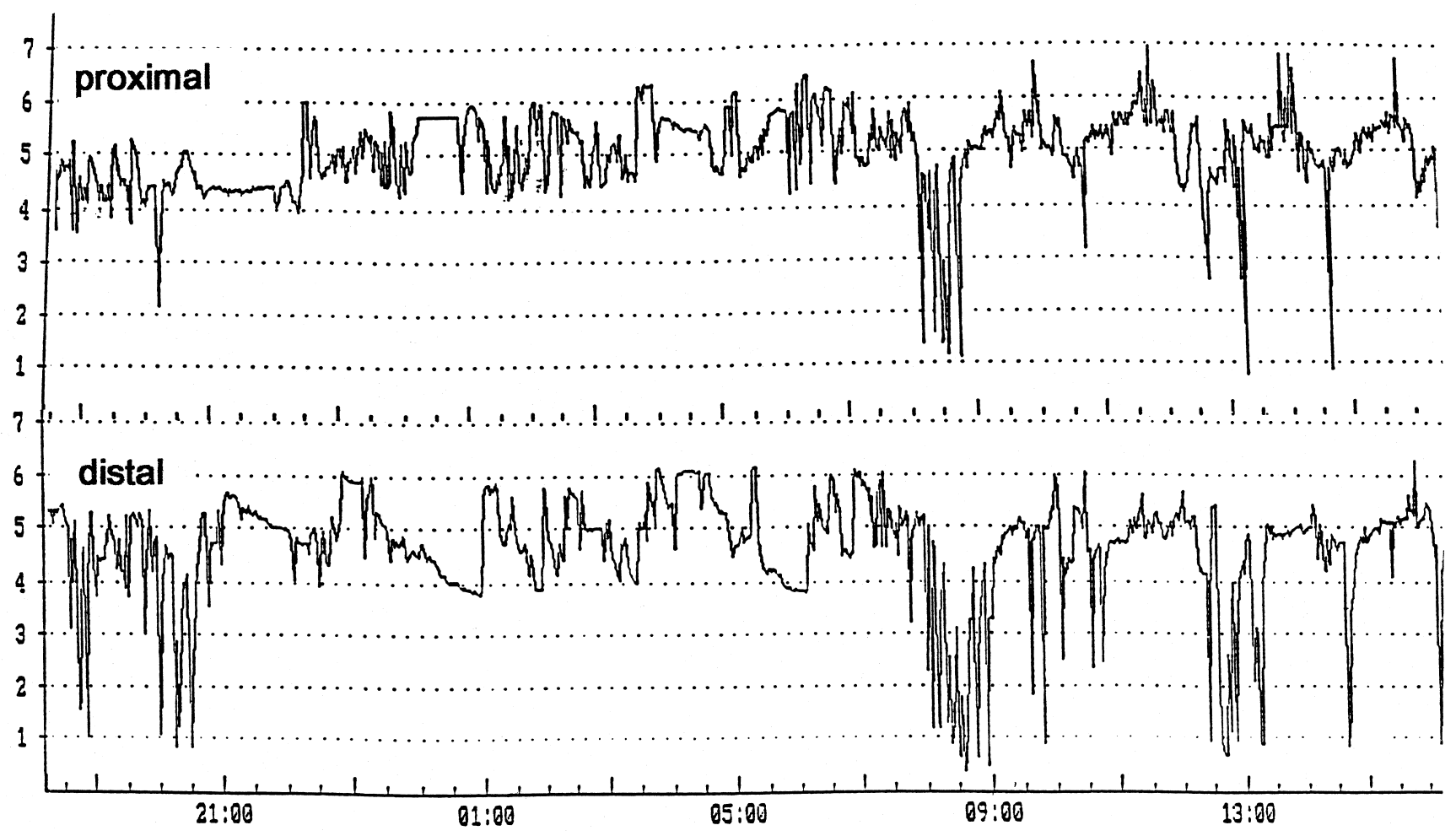

Abb. 4 pH-Metrie-Verlauf über 24 Stunden bei Pat. 5 (proximaler und distaler Messpunkt). Es werden proximal 35 Refluxe gemessen, von denen 2 länger als 5 Minuten andauern, das längste Ereignis wird mit 43 Minuten registriert. Während 4,8\% der Messzeit wird ein pH-Wert von 4 unterschritten. Am distalen Sensor werden 45 Refluxereignisse gemessen, von denen 9 länger als 5 Minuten andauern. Das längste Ereignis ist auch hier 43 Minuten. 13,4\% der Messzeit wird ein pH-Wert von 4 unterschritten.

Die Therapie des Kindes mit Omeprazol führte zu einer völligen Anfallsfreiheit. Wenngleich der inspiratorische Stridor klinisch für das Vorliegen einer VCD spricht, ist bei diesem Patienten nicht auszuschließen, dass eine „klassische“ refluxassoziierte laryngeale und pulmonale Problematik vorliegt. Eine subglottische Schwellung könnte für die inspiratorische Symptomenkomponente verantwortlich sein, die refluxinduzierte bronchiale Hyperreaktivität für die asthmatischen Beschwerden.

\section{Diskussion}

Wie auch schon bei anderen Autoren $[8,10,20])$ überwiegen in den hier vorgestellten Fällen ebenfalls die Mädchen und die Patienten rekrutieren sich überwiegend aus dem peripubertären Altersegment. Eine VCD kann jedoch durchaus auch bereits im Kleinkindesalter (Patient 3 ) und auch bereits im Säuglingsalter auftreten [12]. Bei den 5 von uns vorgestellten Patienten leidet nur 1 Kind koinzident an allergischem Asthma.

Wir fanden bei allen fünf von uns während der letzten 3 Jahre diagnostizierten Patienten ein deutliches pathologisches Refluxmuster am proximalen, im oberen Ösophagusdrittel platzierten Messpunkt. Die klassische gastroenterologische Messposition ergab überwiegend grenzpathologische Befunde, so dass eine diagnostische Überlegenheit der 2-Punkt-pH-Metrie im Zusammenhang mit refluxassoziierten laryngealen Erkrankungen diskutiert werden muss. Inwieweit ein pathologisches Refluxgeschehen im Zusammenhang mit der Erkran- kung als kokausal gesehen werden muss, ist jedoch im Rahmen einer kasuistischen Zusammenstellung schwer zu beurteilen. In einem Fall führte der Einsatz von Omeprazol durch ein Plazebo ebenso zu einer deutlichen Verbesserung der Situation, wie die vorherige Medikamentengabe. Ein anderes Kind wurde durch die alleinige Gabe von Omeprazol anfallsfrei. Zusammenhänge von entzündlichen Erkrankungen des Larynx - insbesondere der Induktion einer Laryngitis durch pathologische Refluxaktivität - wurden mehrfach beschrieben [21-25]. Es stellt sich damit die Frage, ob der pathologische Reflux eine laryngeale Hyperreaktivität auslösen kann und so möglicherweise zum Wegbereiter einer paradoxen Stimmbandreaktion wird. Pathophysiologisch könnte die VCD durchaus als Fehl- bzw. Überfunktion des laryngealen Schutzreflexes bei drohender Aspiration interpretiert werden. Zumindest sollte - insbesondere auch auf dem Hintergrund der frustrierenden therapeutischen Optionen der Refluxproblematik vermehrte Aufmerksamkeit im Zusammenhang mit dem Phänomen einer paradoxen Stimmbandadduktion gewidmet werden.

Auch bei unseren Patienten wird der schon vielfach vorbeschriebene psychogene Hintergrund der Erkrankung deutlich. Es zeigen 3 der 5 Kinder Auffälligkeiten (Überforderung, Schulversagen, Ablöseprobleme), die Krankheitsbezug haben könnten. Diskutiert in diesem Zusammenhang werden z.B. depressive Krankheitsbilder, Angst vor Schulversagen, ein Hang zum Perfektionismus, Störungen des Selbstwertgefühls und auch peripubertäre Ablösungsprobleme $[15,16,20]$. An- 
Tab. 1 Normwerte der 2-Punkt-pH-Metrie bei Kindern

\begin{tabular}{lll}
\hline & proximal $^{\#}$ & distal $^{*}$ \\
\hline Anzahl Refluxe/24 h & $<13$ & $<36$ \\
Anzahl Refluxe $>5 \mathrm{~min} / 24 \mathrm{~h}$ & $<1$ & $<6$ \\
längster Reflux (min) & $<3$ & $<7$ \\
Fraction-time $(\%)$ & $<1$ & $<7$
\end{tabular}

$\#=$ Lit. 27; ${ }^{*}=$ Lit. 28, 29; Fraction-time = prozentualer Anteil der Messzeit mit $\mathrm{pH}<4$

dere Autoren weisen aber darauf hin, dass Untersuchungen an Asthmapatienten eine ebenso hohe Frequenz dieser eher banalen psychischen Abweichungen gezeigt haben $[2,19]$. Es wird damit schwer, einen spezifischen Zusammenhang zwischen bestimmten psychischen Mustern und der Erkrankung VCD zu formulieren.

Im Gegensatz zu der berichteten hohen Koinzidenz allergischer bzw. asthmatischer Erkrankungen mit der VCD wurde von unseren Kindern nur ein Mädchen mit gleichzeitig vorhandenen Allergien identifiziert. Bei einem weiteren Kind mit Sensibilisierungen gegen Erle und Birke konnte eine allergene Relevanz eindeutig ausgeschlossen werden. Es empfiehlt sich daher die Durchführung von spezifischen Provokationsuntersuchungen zur Klärung der allergenen Relevanz von im Haut- oder RAST-Test ermittelten Sensibilisierungen.

Man muss bei der VCD sicherlich von einem multifaktoriellen Geschehen ausgehen, bei dem möglicherweise erst das un- günstige Ineinandergreifen mehrerer Faktoren zu der sich dann präsentierenden Symptomatik führt. Therapeutisch muss daher immer ein individuelles Vorgehen entsprechend den erkannten oder im Vordergrund stehenden Teilursachen entwickelt werden.

Therapeutische Optionen im Anfall sind die Gabe von Heliox, einer Mischung aus 20\% (bzw. 30\%) Sauerstoff und 80\% (bzw. $70 \%)$ Helium [8] und die lokale Applikation von XylocainSpray. Auch einfache Atemübungen sind in der Lage, einen Anfall zu durchbrechen. Positiv beschrieben ist darüber hinaus die Sedierung der Patienten mit z.B. Midazolam und auch durch Injektionen von Botulinum-Toxin werden Erfolge berichtet. Invasive Maßnahmen wie die Intubation oder die Tracheotomie stehen am Ende der therapeutischen Palette und sollten möglichst auf der Basis objektiver Kriterien (Blutgasanalyse) indiziert werden [26]. Die Dauertherapie basiert auf der Beseitigung von potenziellen Triggern (Reflux, Allergie, antientzündliche Therapie, antibiotische Therapie), Entspannungsübungen, Sprachtherapie, Psychotherapie und Biofeedbackverfahren. Sinnvoll ist sicherlich auch eine umfangreiche Aufklärung und Information der Patienten. Als angstreduzierend wird immer wieder die Videodemonstration des Befundes empfohlen $[1,10,15]$.

Auch bei der Nutzung aller zur Verfügung stehenden Maßnahmen ist die Rezidivrate hoch und die Patienten sind immer in der Gefahr, durch antiasthmatisch ausgerichtete Nofallmaßnahmen Schaden zu erleiden. Vorgebeugt werden kann dieser Gefahr durch einen Notfall-Interventionsplan, den der Patient immer bei sich tragen sollte (Abb. 5, Tab.1 -3)

Tab. 2 Synopsis der Befunde der Patienten 1-5

\begin{tabular}{|c|c|c|c|c|c|}
\hline & $\begin{array}{l}\text { Pat. } 1 \\
9 \text { Jahre }\end{array}$ & $\begin{array}{l}\text { Pat. } 2 \\
13 \text { Jahre }\end{array}$ & $\begin{array}{l}\text { Pat. } 3 \\
2 \text { Jahre }\end{array}$ & $\begin{array}{l}\text { Pat. } 4 \\
11 \text { Jahre }\end{array}$ & $\begin{array}{l}\text { Pat. } 5 \\
12 \text { Jahre }\end{array}$ \\
\hline Sensibilisierungen & Erle, Birke & Birke, Milbe & keine & keine & keine \\
\hline Allergien & keine & Birke, Milbe & keine & keine & keine \\
\hline Asthma & nein & ja & nein & nein & nein \\
\hline Laryngoskopie/Bronchoskopie & Bronchitis & o. B. & $\begin{array}{l}\text { Laryngitis/ } \\
\text { Tracheomalazie }\end{array}$ & Laryngomalazie & nicht durchgeführt \\
\hline obere Magen-Darm-Passage & o.p.B. & nicht durchgeführt & Reflux & Reflux & Reflux \\
\hline $\mathrm{pH}$-Metrie proximal & pathologisch + & pathologisch + & pathologisch ++ & pathologisch ++ & pathologisch ++ \\
\hline pH-Metrie distal & pathologisch $(+)$ & pathologisch $(+)$ & pathologisch + & pathologisch ++ & pathologisch ++ \\
\hline $\begin{array}{l}\text { Magenentleerung } \\
\text { (Szintigraphie) }\end{array}$ & normal & normal & nicht durchgeführt & normal & normal \\
\hline Psyche & auffällig & auffällig & nicht untersucht & unauffällig & auffällig \\
\hline Sinusitis & nein & nein & nein & nein & nein \\
\hline
\end{tabular}

\begin{tabular}{|c|c|c|c|c|c|}
\hline & $\begin{array}{l}\text { Pat. } 1 \\
9 \text { Jahre }\end{array}$ & $\begin{array}{l}\text { Pat. } 2 \\
13 \text { Jahre }\end{array}$ & $\begin{array}{l}\text { Pat. } 3 \\
2 \text { Jahre }\end{array}$ & $\begin{array}{l}\text { Pat. } 4 \\
11 \text { Jahre }\end{array}$ & $\begin{array}{l}\text { Pat. } 5 \\
12 \text { Jahre }\end{array}$ \\
\hline Anzahl Refluxe & $16 / 76$ & $43 / 56$ & $24 / 35$ & $31 / 234$ & $34 / 45$ \\
\hline $\begin{array}{l}\text { Anzahl Refluxe } \\
>5 \text { Minuten }\end{array}$ & $2 / 4$ & $0 / 0$ & $3 / 4$ & $0 / 5$ & $2 / 9$ \\
\hline längster Reflux (min) & $14 / 14$ & $3 / 5$ & $76 / 76$ & $4 / 12$ & $43 / 43$ \\
\hline Fraction-time (\%) & $2,2 / 4,4$ & $1,3 / 4,8$ & $26,7 / 26,9$ & $1,6 / 12,9$ & $4,8 / 13,4$ \\
\hline
\end{tabular}

Tab. 3 Ergebnisse der 2-Punkt-pH-Metrie (proximal/distal); pathologische Werte in Fettdruck 


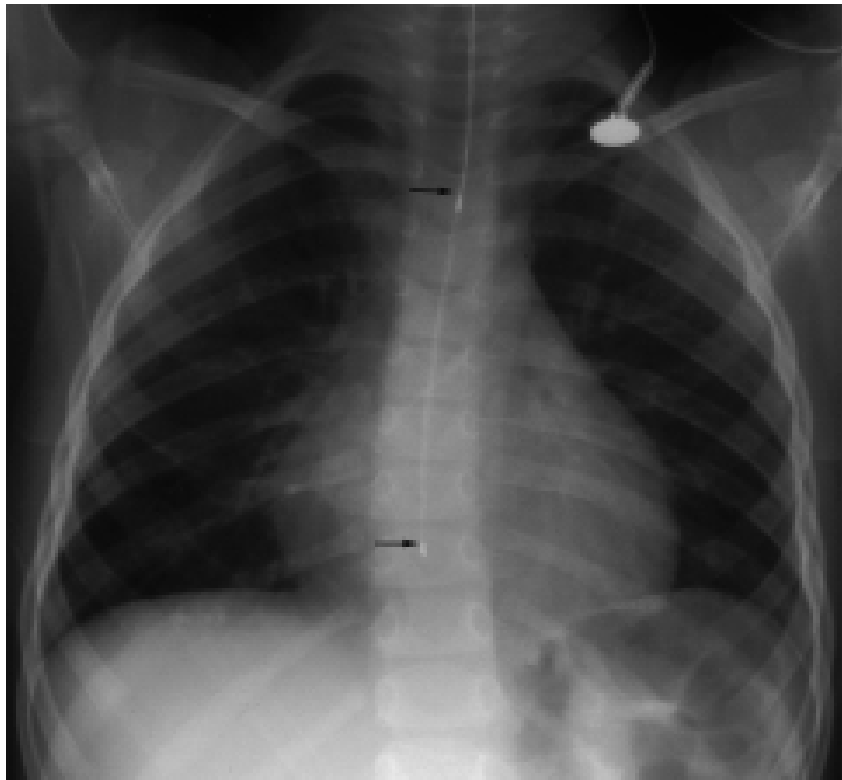

Abb. 5 Sondenposition der Zweipunkt-pH-Metrie. Die Pfeile markieren die Lage der proximalen und der distalen Messelektrode. Die Sonde wurde zur exakten Platzierung des proximalen Messpunktes nach dieser Röntgenkontrolle nochmals $1 \mathrm{~cm}$ nach kranial gezogen.

\section{Literatur}

${ }^{1}$ Niggemann B, Paul K, Keitzer R, Wahn U. Vocal cord dysfunction in three children - misdiagnosis of bronchial asthma? Pediatr Allergy Immunol 1998; 9: 97-100

${ }^{2}$ Christopher KL, Wood RP et al. Vocal cord dysfunction presenting as asthma. N Engl J Med 1983; 308: 1566 - 1570

${ }^{3}$ Meltzer EO, Orgel HA et al. Vocal cord dysfunction in a child with asthma. J Asthma 1991; 28 (2): 141 - 145

${ }^{4}$ Mobeireek A, Alhamad A et al. Psychogenic vocal cord dysfunction simulating bronchial asthma. Europ Respir J 1995; 8: 1978 - 1981

${ }^{5}$ Kenn K, Schmitz M. „Vocal cord dysfunction“ (VCD), eine wichtige Differentialdiagnose zum schweren implausiblen Asthma bronchiale. Pneumologie 1997; 51: $14-18$

${ }^{6}$ Newmann KB, Mason UG, Schmaling KB. Clinical features of vocal cord dysfunction. Am J Respir Crit Care Med 1995; 152: $1382-1386$

${ }^{7}$ Morris MJ, Deal LE, Bean DR et al. Vocal cord dysfunction in patients with external asthma. CHEST 1999; 116/6: 1676-1682

${ }^{8}$ Rutt T, Mansfeld HJ. Vocal cord dysfunction bei jugendlichen Asthmapatienten. Monatsschr Kinderheilk 1999; 147: 817-822

${ }^{9}$ Geist R, Tallet SE. Diagnosis and management of psychogenic stridor caused by conversion disorder. Pediatr 1990; 86: 315 - 317

10 Landwehr LP, Wood RP et al. Vocal cord dysfunction mimicking exercise-induced bronchospasm in adolescents. Pediatrics 1998; $971-974$

${ }^{11}$ Gavin LA, Wambold M, Brugman S et al. Psychological and family characteristics of adolescents with vocal cord dysfunction. J Asthma 1998; 35: 409-417

${ }^{12}$ Heatley DG, Swift E. Paradoxical vocal cord dysfunction in an infant with stridor and gastroesophageal reflux. Oto Rhino Laryngology 1996; 34: 149-151

${ }^{13}$ Nastasi KJ, Howard DA et al. Airway fluoroscopic diagnosis of vocal cord dysfunction syndrome. Ann Allergy, Asthma, Immunology 1997; 78: 586-588
${ }^{14}$ Elshami AA, Tino G. Coexistent asthma and functional upper airway obstruction. CHEST 1996; 110: $1358-1361$

${ }^{15}$ Goldman J, Muers M. Vocal cord dysfunction and wheezing Thorax 1991; 46: 12401 - 12404

${ }^{16}$ Selner JC, Staudenmayer H et al. Vocal cord dysfunction: The importance of psychologic factors and provocation challenge testing. J Allergy Clin Immunol 1987; 79: 726-733

${ }^{17}$ Sette L, Panjo-Ferrara F et al. Vocal cord dysfunction in an asthmatic child: Case Report. J Asthma 1993; 30 (5): 407-412

${ }^{18}$ Reisner C, Nelson HS. Vocal cord dysfunction with nocturnal awakening. J Allergy Clin Immunol 1997; 99: 843 - 846

${ }^{19}$ Logvinoff MM, Lau KY et al. Episodic stridor in a child secondary to vocal cord dysfunction. Pediatr Pulmonol 1990; 9: 46-48

${ }^{20}$ Alpert SE, Dearborn DG et al. To the Editor: On vocal cord dysfunction in wheezy children. Pediatric Pulmonol 1991; 10: $142-143$

21 Jacob P. Proximal eosphageal pH-metry in patients with „refluxlaryngitis“. Gastroenterology 1991; 100: 305-310

${ }^{22}$ Cherry J. Pharyngeal localizations of symptoms of gastroeosphageal reflux. Ann Otol laryngol 1970; 79: 912 -915

${ }^{23}$ Wiener G. Chronic hoarseness secondary to gastroesophageal reflux disease. Am J Gastroenterol 1989; 84: 1503-1508

${ }^{24}$ Shaker P. Esophageal distribution of refluxed gastric acid in patients with reflux laryngitis. Gastroenterology 1995; 109: $1575-1583$

${ }^{25}$ Katz P. Ambulatory esophageal and hypopharyngeal pH-monitoring in patients with hoarseness. Am J Gastroenterol 1990; 85: $38-40$

${ }^{26}$ Cunningham MJ, Eavey RD et al. Familial vocal cord dysfunction. Pediatrics 1984; 76: 750-753

${ }^{27}$ Ahrens P, Heller K. Gastroösophagealer Reflux im Kindesalter. In: Fuchs KH, Thiede A (Hrsg.). Gastroenterologische Funktionsdiagnostik. Springer, 1996: 614-661

${ }^{28}$ Vandenplas Y, Sacre L, Loeb H. pH monitoring in children. Neth J Med 1989; 34: 62 - 73

${ }^{29}$ Euler AR, Byrne WJ. Recurrent pulmonary disease in children: a complication of gastroesophageal reflux. Pediatr 1979; 63: 47 - 51

\section{PD Dr. P. Ahrens}

Zentrum für Kinderheilkunde und Jugendmedizin Klinikum der J. W. Goethe-Universität Theodor-Stern-Kai 7 60590 Frankfurt 